Les attentats du 13 novembre en direct à la télévision : mise en récit de l'événement et de ses ramifications

The November $13^{\text {th }}$ terror attacks live on television: the narratives of an event and its ramifications

Los atentados del 13 de noviembre en vivo en la televisión: las narraciones del evento y de sus ramificaciones

Pierre Lefébure, Émilie Roche et Claire Sécail

\title{
OpenEdition
}

Journals

Édition électronique

URL : https://journals.openedition.org/mots/23766

DOI : $10.4000 /$ mots. 23766

ISSN : $1960-6001$

Éditeur

ENS Éditions

Édition imprimée

Date de publication : 5 novembre 2018

Pagination : $37-57$

ISBN : 979-10-362-0082-3

ISSN : 0243-6450

Référence électronique

Pierre Lefébure, Émilie Roche et Claire Sécail, « Les attentats du 13 novembre en direct à la télévision : mise en récit de l'événement et de ses ramifications », Mots. Les langages du politique [En ligne], 118| 2018, mis en ligne le 05 novembre 2020, consulté le 22 avril 2022. URL : http://

journals.openedition.org/mots/23766; DOI : https://doi.org/10.4000/mots.23766 


\section{Les attentats du 13 novembre en direct à la télévision : mise en récit de l'événement et de ses ramifications}

Le 13 novembre 2015, à 22h59, sept minutes après le début de l'édition spéciale de LCI-TF1, la présentatrice lance : «C'est horrible, on a l'impression de revivre un peu ce qu'on avait vécu au moment des attentats de janvier. Cette fois, je crois que c'est clair, on parlera des attentats du 13 novembre 2015 ». Cette capacité à nommer l'événement, alors que son étendue et sa portée sont à peine appréhendées, et à le sérialiser, en le situant par rapport à des événements antérieurs de même nature, montre que ce qui vient de survenir ce 13 novembre se produit dans une société qui se conçoit et se perçoit déjà comme une société post-attentats, capable, au-delà du choc, de faire fonctionner des mécanismes discursifs et des catégories de sens pour traiter l'événement et y faire face aussi bien cognitivement que normativement et émotionnellement.

Au cours des années 2000 , selon le baromètre de la menace terroriste de l'IFOP, un Français sur deux en moyenne considérait la menace terroriste comme "élevée "1. Après l'affaire Merah en 2012, ce ressenti touche désormais les trois quarts des enquêtés. Mais le taux bondit vraiment après les attentats de janvier 2015, quand il devient supérieur à $90 \%$. Surtout, la répartition entre ceux qui, parmi les enquêtés, pensent que la menace terroriste est «très élevée » et ceux qui pensent qu'elle est seulement «élevée » - jusqu'alors majoritaires - s'inverse pour la première fois². Initié en 2001 après le 11-Septembre, ce baromètre de la menace terroriste renvoie l'image d'une société française qui, à partir de 2012, semble avoir intégré majoritairement l'idée de devoirvivre avec une menace terroriste aiguë et permanente.

1. À l'exception de deux «pics» après le 11-Septembre (64\% en octobre 2001$)$ et les attentats de Madrid (60\% en septembre 2004). Source : Le baromètre de la menace terroriste - vague 39, IFOP/Atlantico, juin 2017.

2. Après janvier 2015 , les premiers représentent $49 \%$ de l'échantillon (contre $18 \%$ en 2014), les seconds $44 \%$. Source : lbid.

Université Paris 13, IRISSO

pierre.lefebure@univ-paris13.fr

Université Paris 3, CIM-ERCOMES

emilie.roche@univ-paris3.fr

CNRS, IRISSO

claire.secail@dauphine.fr 
Les discours post-attentats ne seraient donc pas tant une conséquence factuelle de l'événement qu'une expression saillante de l'état de la société et du débat public au moment où il survient, et qui lui préexiste donc. En ce sens, il convient d'interroger leur rapport à « un ensemble de cadres prédiscursifs collectifs (savoirs, croyances, pratiques) qui donnent des instructions pour la production et l'interprétation du sens en discours »(Paveau, 2006, p. 118). Les premières heures de l'attentat sur les chaînes de télévision nous servent en cela de point d'observation pour établir dans quelle mesure le traitement médiatique de l'événement abrite un ensemble de données antérieures à sa mise en discours. L'objectif est d'examiner selon quelles modalités discursives se manifestent les premiers mécanismes de réflexivité concomitamment à l'effet de sidération. Témoignages d'anonymes, duplex des envoyés spéciaux, analyses des experts, commentaires des journalistes : comment l'expression d'une conversation sociale sur les attentats émerge-t-elle et quelles orientations prend-elle?

Pour répondre à cette question, nous avons travaillé sur un corpus d'une vingtaine d'heures d'éditions spéciales de TF1, France 2, France 3, M6, Arte et BFM TV datant des 13 et 14 novembre $2015^{3}$. Ce choix de s'intéresser aux discours médiatiques les plus précocement énoncés 4 permet de traiter des données qui, si elles restent inscrites dans des pratiques rédactionnelles routinières marquées par la primauté des sources institutionnelles, ne sont pas encore susceptibles d'être orientées par les entreprises de construction du sens de l'événement que les autorités publiques et acteurs politiques cherchent à produire (Altheide, 2009, 2017 ; Frau-Meigs, 2005 ; Garcin-Marrou, 2001). Par ailleurs, en nous attachant à des discours énoncés en situation de retransmission télévisée en direct aussi bien par des reporters de terrain et des témoins que par des journalistes spécialisés et des experts en plateau, nous minimisons aussi la capacité de réflexivité de leurs énonciateurs que ménagerait beaucoup plus l'écrit.

D'un point de vue méthodologique, ce corpus est travaillé à partir d'une analyse de contenu thématique, mise en perspective avec l'examen des dispositifs télévisuels, mais aussi d'analyses discursives basées sur la terminologie, le lexique et le cotexte désignant la situation, et d'une analyse argumentative faisant apparaître des phénomènes de dissensus/consensus dans les discours. On peut ainsi observer comment la chronologie et les catégories qui s’y rattachent (acteurs, thèmes, dispositifs, normes) contiennent les sédiments de traitements antérieurs et à venir, et mesurer jusqu'à quel point les choix de

3. Cette étude est menée dans le cadre du projet SENSI-TV-T de l'équipe LCP-IRISSO, qui a obtenu le soutien du CNRS (Appel Attentats-Recherche) en 2016.

4. Les discours médiatiques liés à ces événements ne se limitent pas à ce premier contexte d'énonciation. Ils peuvent aussi être considérés, du point de vue de leur accumulation au fil du temps, comme susceptibles de former ce qui a pu être interprété à propos des attaques du 11-Septembre comme un «disaster marathon " (Blondheim, Liebes, 2003) distinct du modèle théorique du «media event». 
dispositifs, de formats et de régimes discursifs mobilisés par les rédactions participent à produire plusieurs cadres interprétatifs du même événement. Ce faisant, il s'agit de discuter ce que peut recouvrir la notion de «discours postattentats » en interprétant la part respective de chacune des perspectives narratives identifiées par nos analyses entre, d'une part, un pôle concentré sur l'exceptionnalité des faits et du contexte constituant un tournant pour la société française et, d'autre part, un pôle concentré sur l'intégration des faits et du contexte à un état déjà présent ou latent qui ne serait pas tant bouleversé que cristallisé dans les événements.

\section{Les récits télévisuels du 13 novembre : une hétérogénéité des discours}

\section{Dispositifs d'édition spéciale et variations thématiques}

Un examen de la morphologie des éditions spéciales permet d'établir dans quelle mesure le dispositif défini par les chaînes a pu structurer différemment le cadre interprétatif de l'événement. Une édition spéciale se repère à travers les efforts humains, techniques et budgétaires déployés par les rédactions pour couvrir en longueur et en direct un événement, mais également à travers les sacrifices commerciaux consentis. Le choix de «casser l'antenne » ou de laisser dérouler la programmation prévue est une première façon de souligner les différentes logiques éditoriales en jeu et d'attester une relative hétérogénéité des discours. Le 13 novembre, BFM TV est la première à interrompre sa programmation dès $21 \mathrm{~h} 52^{5}$. Le présentateur de la tranche prend l'antenne sans attendre la coupure publicitaire : "Bonsoir à tous. Bienvenue dans Week-end Direct sur BFM TV. Nous prenons l'antenne un petit peu plus tôt que prévu parce qu'il s'est passé quelque chose ce soir dans le $10^{\mathrm{e}}$ arrondissement de Paris. C'est l'information de la soirée. "Suivront France 3 à 22 h50 dans le cadre des titres du Soir 3 Week-end, puis TF1 à 22h52, après la diffusion du match de football France-Allemagne ("La France s’impose 2-o. Malheureusement, il y a eu de terribles attentats en France, Bixente. On va tout de suite rendre l'antenne», annonce le commentateur sportif) et, plus tardivement, France 2 à 23 h55 en pleine allocution du président de la République, cette dernière marquant un tournant de la soirée puisqu'elle authentifie la nature terroriste de l’événement. En revanche, Arte et M6 n'ayant ni la mission, ni la capacité d'assurer le suivi

5. Dès 21h35, sur ìTélé, un bandeau «Dernière minute» annonce : «Une brasserie à proximité du Stade de France vient d'exploser, faisant au moins un blessé ». À 21h50, les commentateurs sportifs réunis pour le match évoquent avec leur reporter sur place «deux énormes déflagrations ». Ils parlent peu après des «fusillades qui se seraient produites », renonçant à traiter le match. La chaîne entre en édition spéciale à 21 h59. 
en direct d'un événement majeur, leurs téléspectateurs ont pu suivre respectivement les Noces de Figaro et les épisodes «Otages » et «Bombes humaines» de la série $N C I S$, entre lesquels M6 diffuse l'allocution présidentielle, en différé à oh58 (soit plus d'une heure après avoir été prononcée).

Si seules les chaînes généralistes TF1, France 2 et France 3 ainsi que les chaînes d'information en continu sont en mesure d'assurer un dispositif d'édition spéciale, celui-ci contraste singulièrement de l'une à l'autre. Après la retransmission du match, TF1 diffuse le canal de LCl, chaîne d'information en continu de son groupe. La plupart des téléspectateurs n'ayant pas accès à cette chaîne alors payante découvrent ainsi des journalistes dont ils ne sont pas familiers en même temps que les premiers éléments d’information. La coopération est plus compliquée dans le cas des deux chaînes du service public : France 3, qui doit assurer la tranche habituelle du Soir 3, y improvise une édition spéciale et, au moment de rendre l'antenne, indique juste que «nos confrères de France 2 prennent le relais à l'heure qu'il est». En cette veille de week-end, période creuse pour l'information télévisée, la rédaction de France 2 a du mal à s'organiser. Faute de journalistes disponibles, la chaîne n'interrompt l'émission de débat Ce soir ou jamais (consacrée au changement climatique) qu'au milieu de l'allocution de François Hollande ${ }^{6}$. Un mauvais raccord technique sonore donne au téléspectateur une idée de l'ambiance en régie : «On est ridicule, là!»

Ces dispositifs ne sont pas sans influencer les mises en récit des attentats au point de structurer la perception hétérogène d'un événement dont l'étendue spatiale (dissémination sur différents sites) et la progression temporelle (chronologie incertaine) échappent largement aux journalistes et, par extension, aux publics. Si les chaînes ont en commun de proposer un récit majoritairement factuel (figure 1), entre 78,1 \% du volume horaire sur TF1 et 91,3\% sur France 2, la répartition des thématiques abordées et le temps d'antenne accordé aux différents sites des attentats fluctuent d'une chaîne à l'autre (figure 2).

Ayant des équipes mobilisées sur place pour assurer la diffusion de l'événement sportif, TF1 consacre ainsi $9 \%$ de son temps aux attaques du Stade de France (contre $1 \%$ sur France 2 et $7 \%$ sur BFM TV). Par un concours de circonstances, le remplaçant occasionnel du Soir 3, qui assistait également au match à titre privé, reprend son engagement professionnel pour effectuer cinq longs duplex avec le présentateur de l'édition spéciale, soit $15 \%$ du temps d'antenne de France 3. TF1 et France 3 sont ainsi les seules chaînes à avoir consacré plus

6. Après l'attentat de Nice (14 juillet 2016), la chaîne s'obligera à prendre rapidement l'antenne pour ne pas rééditer le scénario du 13 novembre, qui avait provoqué de nombreux reproches au sein de la rédaction. "Notre difficulté est justement de ne pas avoir de chaîne d'info, avec des équipes en permanence et des procédures adaptées » expliquera le directeur de la rédaction de France TV (Le Monde, 15 juillet 2016). Deux mois plus tard, le groupe lancera la chaîne France Info sur la TNT. 


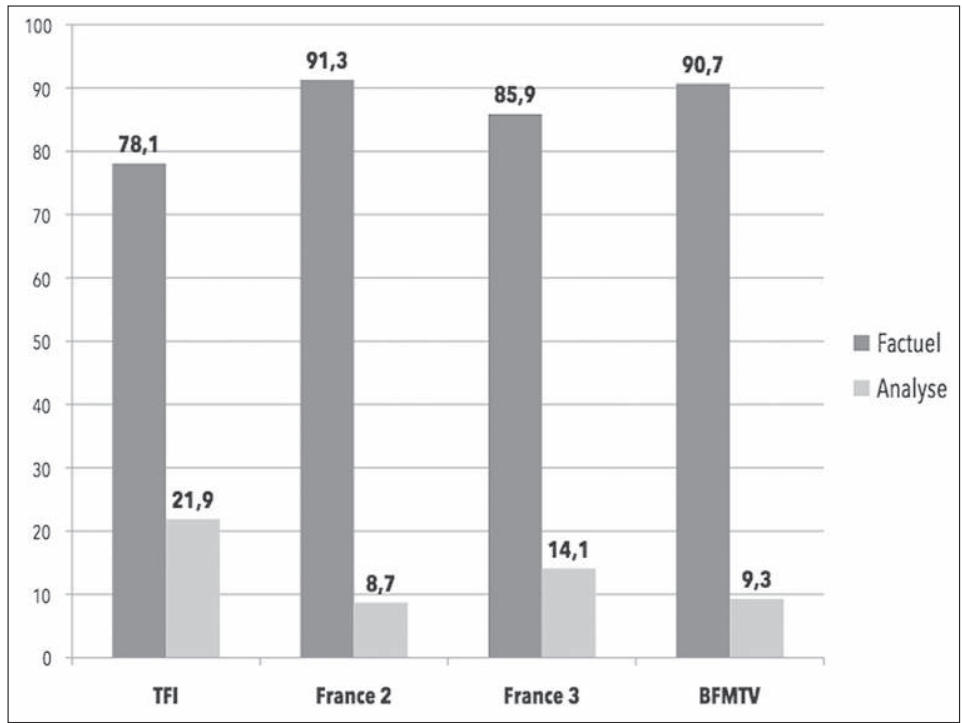

Figure 1. Mode de traitement de l'événement dans les éditions du 13 novembre (\% du temps d'antenne)

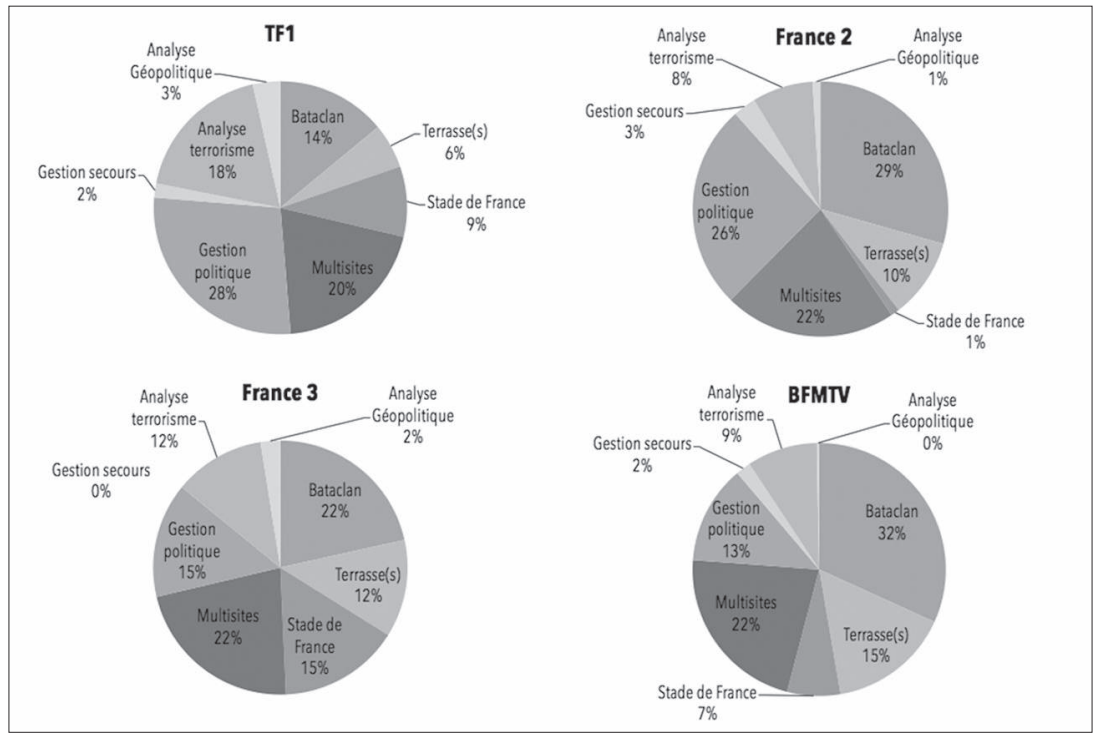

Figure 2. Répartition thématique des éditions du 13 novembre (\% du temps d'antenne) 
de temps aux attaques du Stade de France qu'à celles des terrasses (6 \% sur TF1 et $12 \%$ sur France 3 ).

En revanche, la situation au Bataclan est la plus abondamment couverte par les quatre chaînes, même si l'amplitude du temps d'antenne varie de plus du double (14\% surTF1, 29 \% sur France 2, 32 \% sur BFM TV). La nature de l'événement et le spectre d'un nombre de victimes élevé expliquent le plus fort intérêt des journalistes pour ce site. À l'heure où l'évacuation du Stade de France est quasiment achevée et alors que les équipes de secours ont déjà pris en charge les sites des terrasses, leur attention se concentre sur la situation boulevard Voltaire, où chaque chaîne envoie plusieurs journalistes en renfort. Plus homogène est la proportion accordée à un traitement «multisite» de l'événement, mode narratif utilisé surtout par les journalistes en plateau lors des séquences de «rappel des événements » et de «bilan des victimes », de plus en plus nombreuses au fil de la soirée.

Si le traitement factuel domine, la part de l'analyse n'est pas négligeable. Celle-ci s'explique d'une part par la présence en plateau des chefs de service Politique et Étranger qui oscillent entre informations factuelles et recul analytique, et d'autre part par les premières prises de parole des experts de la sécurité et du renseignement. Ces dernières interviennent avant même l'allocution présidentielle sur France 3 (23h18) et TF1 (23h31), qui consacrent proportionnellement plus de temps à analyser les enjeux terroristes et géopolitiques que leurs concurrentes (14,1 \% pour France 3 et $21,9 \%$ pour $\mathrm{TF}_{1}$, contre $8,7 \%$ pour France 2 et $9,3 \%$ pour BFM TV). La parole experte arrive plus tardivement sur France 2 (oh59) et BFM TV (1h26).

La place accordée à la parole des témoins des différents sites d'attentats est un dernier indicateur de l'hétérogénéité des dispositifs et des cultures, sinon journalistiques, du moins éditoriales (figure 3). TF1 est la chaîne qui fait le moins usage de cette parole rapportée à forte charge émotionnelle, à hauteur de 2,2 témoignages en moyenne par heure. Àl'inverse, la culture du témoignage sur BFM TV, plus institutionnalisée?, conduit la chaîne à mobiliser souvent cette parole : en moyenne, 4,8 témoins par heure viennent mettre en mots les réalités insoutenables que ni les comptes rendus ni les images des reporters ne peuvent attester.

\section{Un processus de nomination différencié de l'événement}

La qualification de l'événement en cours varie d'une chaîne à l'autre en fonction de l'heure de la prise d'antenne, mais également en fonction des précautions oratoires adoptées par les différentes rédactions. Une chronologie sémantique

7. La chaîne diffuse des appels à témoignages et a mis en place un site dédié pour que les anonymes puissent télécharger leurs vidéos. 


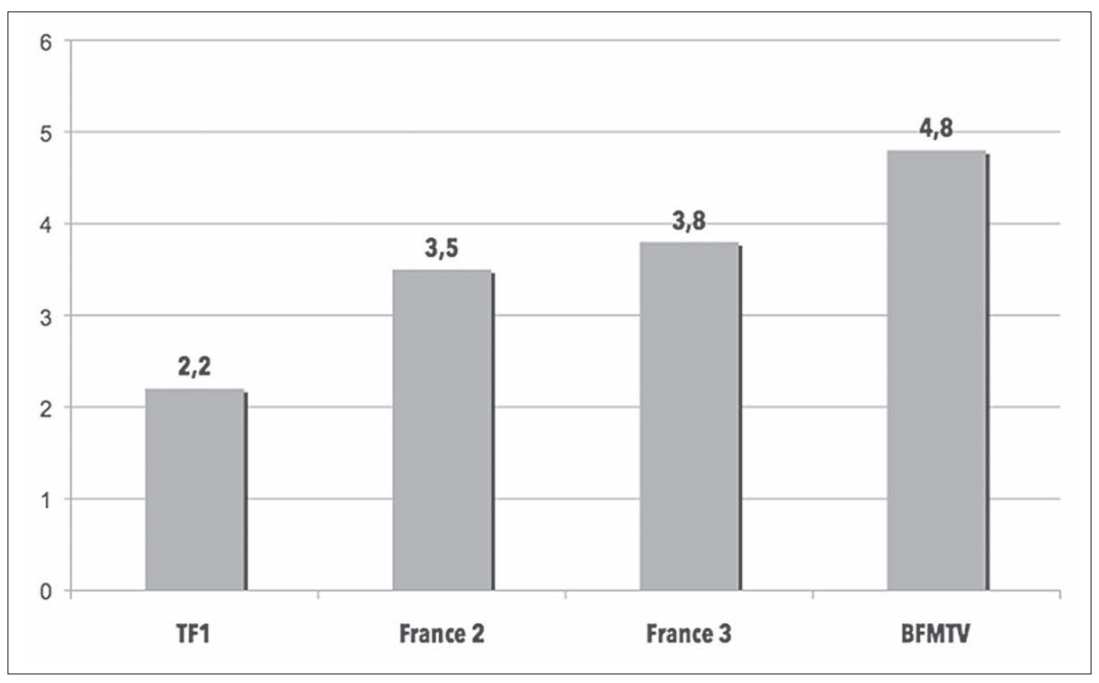

Figure 3. Nombre moyen de témoignages/heure

s’appuyant sur les principales désignations de l'événement à partir des items «attentat», «terrorisme-iste », « djihadisme-iste » (figure 4) permet de cerner ce processus à forts enjeux. Les premières qualifications d'un événement violent, celles qui s'opèrent en direct à la télévision, en fixent en effet durablement la mise en sens, l'interprétation, et les implicites sociaux et politiques (GarcinMarrou, 2001) - «l'acte de nommer» (Calabrese Steimberg, 2012) engageant une responsabilité partagée des journalistes. Ainsi BFM TV propose dès $21 \mathrm{~h} 57$ de classer l'événement dans la catégorie sémantique de l' "attentat» et de l'«attaque terroriste » tout en restant dans un conditionnel énonciatif de prudence. TF1 affirme d'emblée la nature des événements en cours à $22 \mathrm{~h}_{52}$ : «il y a eu de terribles attentats en France ». Neuf minutes plus tard, France 3 postule la survenue d' "attentats» et d' "attaques terroristes » puis France 2 l'affirme à 23 h57 dès sa prise d'antenne. Le moment permettant aux journalistes de se délester de leurs précautions oratoires est celui du discours du président de la République qui qualifie clairement l'événement et valide à la fois sa nature et l'emploi d'un champ sémantique référencé comme étant celui du «terrorisme» et de l' "attentat».

L'analyse de la terminologie employée le 13 novembre au soir permet de repérer 9 items lexicaux communs : «attaques», «attentats», «fusillade(s)», «explosion(s) », « situation», «terrorisme-iste», «terreur», «prise d'otages». On remarque que coexistent des termes génériques englobant l'ensemble de l'événement («attentats», «attaques», «terrorisme», «terreur» et "situation») et des termes beaucoup plus précis quant à la nature des actions en cours («fusillades», 


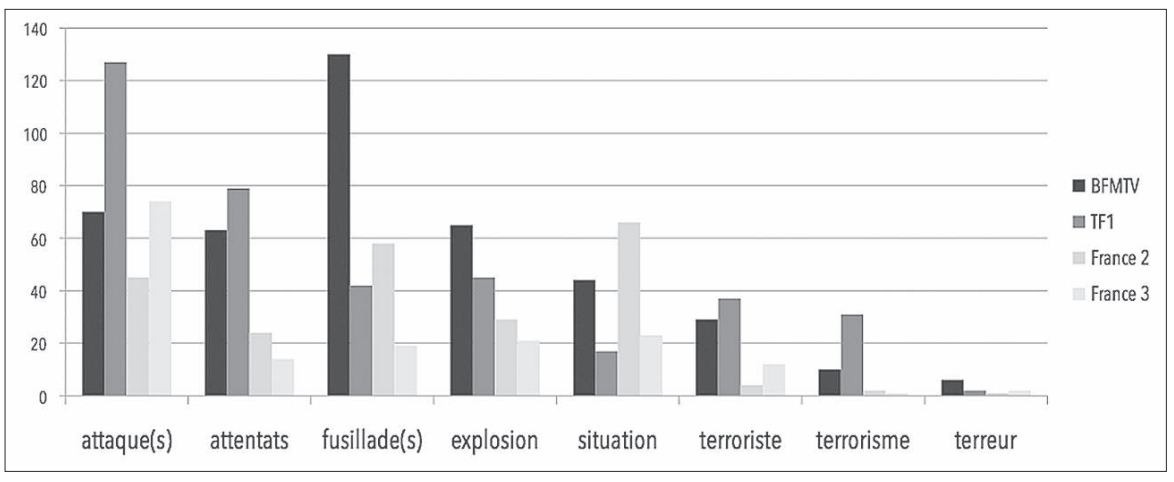

Figure 5. Fréquence d'emploi des termes utilisés pour désigner l'événement (nombre)

les chaînes, le cotexte de l'item «attaque » est celui de la planification, de la synchronie et de la quantité : "attaques coordonnées», "attaques simultanées », "attaques multiples». Ici les cotextes sont d'ordre descriptif, ils ne font pas référence à une situation préconçue ou anticipée. En revanche, les cotextes de «situation » arbitrent des effets de sens précis et porteurs d'enjeux sociétaux : "situation nouvelle», "situation exceptionnelle», "situation inédite », "situation chaotique», "situation critique », "situation de guerre ». La conséquence des «attaques» se traduit d'abord par un effet de surprise et de sidération qui glisse au fur et à mesure de la soirée, et notamment après l'intervention du chef de l'État, vers une nomination de l'événement lourde d'implicites : la «guerre ». Ajoutons que BFM TV se distingue des autres chaînes en inscrivant les attaques dans un régime de valeurs qui dépasse largement le seul cadre des événements en cours : «attaque contre l'humanité », «attaque contre nos valeurs». Enfin, les cotextes du nom "attentat» insèrent l'événement dans une sérialité sur laquelle nous reviendrons. Ainsi la « vague d'attentats » est un «nom événement» qui intègre le 13 novembre dans une série débutée en janvier 2015, probablement non close, avec des attentats à venir (le mot «vague » impliquant un mouvement de flux/reflux laissant présager la répétition). Plus largement, les éléments prédiscursifs des énoncés télévisuels du 13 novembre constituent une injonction consensuelle à se figurer une continuité des attentats sur le sol français et suggèrent aux citoyens d'accepter les mesures fermes prises par l'autorité politique.

Dès le 14 novembre, on observe une normalisation des dispositifs télévisuels. Les éditions spéciales du lendemain renouent avec des modes ordinaires de mise en récit de l'information télévisée : maillage étendu des journalistes sur le terrain, reportages plus synthétiques et construits que la veille, plateaux d'invités étoffés, etc. Hors service le 13, M6 diffuse une édition spéciale dès 8 h45, à l'inverse d'Arte qui ne bouleverse pas ses programmes 
habituels. Normalisés qualitativement, les dispositifs d'éditions spéciales du 14 novembre restent exceptionnels sur un plan quantitatif pour des chaînes généralistes (tableau 1$)^{8}$. Ce phénomène rapide de normalisation, qui inscrit l'événement dans deux temporalités distinctes, a dans une certaine mesure déjà été observé après le 11-Septembre, quand les récits médiatiques de l'attentat articulaient un présent immédiat en temps réel (processus d'identification des victimes, traque des terroristes, etc.) et un présent historique inscrivant déjà l'événement dans le passé (Scannell, 2006).

Tableau 1. Éditions spéciales des chaînes généralistes le 14 novembre

\begin{tabular}{l|c|c|c|c|c}
\hline & TF1 & France 2 & France 3 & Arte & M6 \\
\hline Durée & $11 \mathrm{~h} 15$ & $13 \mathrm{~h} 35$ & $5 \mathrm{~h} 30$ & 0h45 & $5 \mathrm{~h} 40$ \\
\hline
\end{tabular}

\section{Discours disponibles et discours encapsulés : les ramifications du récit}

L'analyse des discours des 13-14 novembre permet d'observer trois processus distincts de ramifications du récit de l'attentat : la mise en séries, la mise en causalités et la mise en interactions dissensuelles/consensuelles.

\section{Sérialisation}

Face au traumatisme et à la suspension de sens induits par l'événement (Arquembourg, 2003), les journalistes, les experts, mais également les témoins utilisent des discours déjà disponibles pour rationaliser, symboliser et produire un récit. Ce «déjà là » des discours peut s’appréhender à travers la notion de cadres prédiscursifs collectifs (Paveau, 2006), mobilisés dès lors qu'un attentat se produit avec des effets de sens qui classent l'événement en train de se dérouler dans une sérialité interprétative. Tôt dans la soirée du 13 novembre, on observe une première sérialisation à travers l'utilisation par les journalistes de BFM TV de référents topographiques. La géographie des «terrasses» rappelle l'attentat de Charlie Hebdo et contribue à classer les événements en direct dans la catégorie «attentat» :

À quelques dizaines de mètres à vol d'oiseau des lieux de l'attentat de Charlie Hebdo [...] à 100, 150 mètres de l'endroit où Ahmed Merabet, l'un des policiers, a été abattu. (Reporter, BFM TV)

8. BFM TV restera en édition spéciale au-delà des trois jours de deuil national. Le bulletin météo sponsorisé revient à l'antenne le 16 novembre à 4 h30 et la première coupure publicitaire réapparaît le même jour à 13h27, après la minute de silence. La mention «Édition spéciale » ne disparaîtra que le 17 à $6 \mathrm{~h}$, après $80 \mathrm{~h}$ d'antenne. 
Ce premier niveau de sérialisation ancre «le 13 novembre» dans une référence $a u$ « 7 janvier», et l'encapsule ainsi dans un système référentiel qui fait sens pour le téléspectateur.

La seconde sérialisation de l'événement est activée à partir des modalités discursives de l'expérience, celle des journalistes (Étienne Leenhardt sur France 2 qui a vécu le 11 septembre 2001 comme correspondant à Washington) comme celle des témoins directs qui se réfèrent spontanément à d'autres attentats pour mettre en mots ce qu'ils vivent. Dans ces récits d'expériencetémoignages, le sens se construit à partir du souvenir d'événements traumatiques et du registre discursif de l'émotion pour déboucher sur la même référence matricielle :

Pour avoir vécu aux États-Unis en 2001 les attentats du 11-Septembre, j'ai envie de tenter ce soir une comparaison. [...] J'ai l'impression que la France, ce soir, cette nuit, est en train de vivre son 11-Septembre. [...] La France va vivre un véritable calvaire. [...] Je me souviens très bien, pour m'être trouvé à Washington [...]. Forcément, ça va nous marquer, ça va marquer nos enfants. (É. Leenhardt sur France 2)

Y avait pas mal d'Américains... Mes enfants vivent même aux États-Unis donc... Certains ont connu le 11-Septembre... Donc après la fusillade, ils ont voulu aller... (Un témoin sur BFM TV)

Cet effet de sérialisation par l'expérience affective et les souvenirs qu'elle évoque demeure cependant marginal au cours de la soirée. Il tranche avec les mises en série opérées par les experts et les journalistes spécialisés qui s'efforcent de maintenir à distance tout marqueur émotionnel et dont les discours produisent un double effet de sens en «encapsulant» l'événement d'une part dans une typologie des modes opératoires terroristes, et d'autre part dans une situation géopolitique internationale.

L'événement encapsulé l'est d'abord à partir de la référence aux attentats de janvier, mais également à partir de l'histoire du terrorisme français et international:

Une séquence qui nous rappelle [...] des attentats de Charlie Hebdo. [...] Souvenezvous de l'Australie [...] Ça rappelle le musée du Bardo. [...] L'arme dont disposait Mehdi Nemmouche au musée juif de Bruxelles [...] C'est l'arme d'Amedy Coulibaly. (BFM TV)

La plupart des pays européens ont connu des attentats [...]. Le plan multi-attentats [...] c'est un plan conçu après les attaques de Bombay [...]. II faut pas oublier que la France est en guerre contre le terrorisme depuis des années, faut pas oublier le terrorisme algérien, ces années de politique en Algérie, 95, Khaled Kelkal. (TF1)

C'est une affaire qui ressemble très largement à ce qu'on a eu à Bombay, avec des commandos mobiles [...] l'attentat raté du Thalys [...]. (France 3) 
Cette mobilisation d'événements antérieurs pour qualifier l'événement présent permet en même temps, d'une façon qui peut sembler paradoxale, d'inscrire le «13 novembre » dans une rupture sans appel, une brèche :

C'est la première fois qu'à Paris, on a ce type d'explosions, avec peut-être des attentats-suicides, un peu comme ceux qui se sont pratiqués à Beyrouth il y a deux jours à peine [...] Équipés comme en Irak, en Syrie, en Afghanistan... comme au Liban. (BFM TV)

Si c'est l'État islamique, c'est une tout autre affaire : c'est une action de guerre [...] il y a une graduation [...] de masse. C'est-à-dire faire le maximum de victimes en un minimum de temps... [...] Un terrorisme de masse, tuer un maximum de gens [...] À Madrid... Atocha... un procédé qui était beaucoup plus classique. (TF1)

La sérialisation dépend in fine essentiellement des locuteurs, de leurs expériences professionnelles ou personnelles, de leurs compétences et plus largement de leur état cognitif et émotionnel au moment où ils s'expriment. Dans tous les cas, elle contribue à façonner le sens social de l'événement.

\section{Interprétation causale}

Au-delà de la description des faits, les éditions spéciales du 13 novembre développent une approche plus interprétative à partir de l'examen du contexte. À peine est-on informé du drame et saisi par l'intensité émotionnelle que l'on est conjointement invité à, voire sommé de considérer froidement des éléments plus généraux. Cette perspective interprétative, qui se retrouve dans les éditions du lendemain midi, mobilise une perspective narrative selon laquelle ce qui s'est passé était connu, au moins de certains, pour devoir survenir plutôt que pouvoir être évité. C'est une sorte de «on le savait», parfois rapporté à des attentats précédents ou aux engagements géostratégiques de la France susceptibles de provoquer des représailles ennemies. Ce « on le savait» n'équivaut pas à un « on vous l'avait bien dit » du fait du caractère restreint des cercles au sein desquels ce qui pouvait être su l'était effectivement ou du fait d'une cécité plus ou moins volontairement entretenue au sein de la société française. Les attentats du 13 novembre viendraient ainsi actualiser aux yeux de la population ce qui était pourtant déjà constitué, imposant une mise au clair brutale sur la nature du contexte dans lequel ils surviennent. On repère deux éléments complémentaires des mécanismes spécifiques de cette construction narrative sur toutes les chaînes.

Le premier est constitué par l'énonciation de ce que ces attentats, pour inédits qu'ils soient dans leur forme en France, relèvent d'une stratégie et de modes d'action connus et prévus pour survenir à brève échéance. Dans la nuit du 13 novembre, cette idée est plus ou moins développée cinq fois sur France 2 (2ho4 d'antenne) et sur France 3 (1h20), dix fois sur BFM TV (4ho7) et dix-neuf fois surTF1 (3h10), par exemple sous les formes suivantes : 
C'est un scénario qui était craint, auquel les services étaient préparés depuis janvier. (Expert, TF1, 23h37)

Tous les interlocuteurs avec qui je discute [...] depuis des mois s'attendaient à une attaque comme celle-ci. C'est-à-dire que s'ils avaient pu l'écrire, ils l'auraient écrit. On savait exactement. (Spécialiste Police, France 2, 1h43)

Le ministère de l'Intérieur ne cesse de répéter, la France est vraiment placée en première ligne [...] On pense aussi à tous ces djihadistes qui sont partis s'entraîner sur le même terrain syrien, qui peuvent représenter une menace à leur retour. (Spécialiste Étranger, France 3, 23ho6)

Les propos sont remarquablement identiques quant au caractère inéluctable des attentats. Or, cette inéluctabilité ne résulte pas du hasard statistique ou d'une fatalité immanente, mais - et c'est le deuxième élément de la construction narrative - d'une logique de conflit présentée comme l'état dans lequel la société française se trouve déjà mais qui ne serait pas suffisamment compris ou admis. Cet état, c'est la guerre dont les attentats déjoués ou commis sont des symptômes plus qu'ils n'ont de signification par eux-mêmes. Récurrent sur TF1, l'énoncé se retrouve aussi sur les autres chaînes :

Le terrorisme, c'est souvent la réponse qui est faite à une guerre. La France est en guerre contre l'État islamique. Les autorités n'ont pas cessé de le proclamer et de le rappeler. (Spécialiste Étranger, TF1, 1hog)

Il faut qu'on arrive à le comprendre une bonne fois pour toutes : nous sommes en guerre. [...] C'est une guerre qui vise des civils, c'est une guerre qui ne définit pas un champ de bataille comme on le conçoit dans les livres d'histoire. (Expert, TF1, 1h35)

On retrouve François Hollande en tant que chef de guerre. [...] La France est en guerre. (Présentateur, France 2, 1h54)

La première fois que l'état d'urgence a été prononcé [...] c'était lors de la guerre d'Algérie, en 1961. Donc véritablement une situation qui est vécue comme une situation de guerre sur le territoire français. (Spécialiste Étranger, France 3, oho5)

Outre ces argumentations spécialisées, plus encore développées le lendemain, plusieurs mécanismes entretiennent la référence à la guerre. La récurrence d'expressions incluant le terme "guerre» ("arme de...», «blessure de...», "scène de...», "théâtre de... ») produit un effet d'évidence factuelle par le lexique. Par ailleurs, le rappel récurrent que l'état d'urgence décrété dans la nuit remonte à une loi adoptée dans le contexte de la guerre d'Algérie et qui n'avait plus été appliquée sur l'ensemble du territoire métropolitain depuis lors produit un effet suggestif. Enfin, la récurrence des images montrant des patrouilles militaires produit une authentification visuelle de la militarisation de la situation, parfois soulignée par les mots des reporters sur le terrain : «les rues sont aux mains de l'armée, ces soldats qui ont disposé des 
poubelles au milieu de cette rue de Charonne. Une sorte de check point pour assurer la sécurité» (France 2, oh23).

La guerre est donc à la fois un constat factuel et un état préalable déjà énoncé, mais tendant à être ignoré et désormais cruellement révélé. Cette articulation s'accentue dans la matinée du 14 novembre autour des déclarations du président de la République, en direct puis en rediffusion («c'est un acte de guerre [...] commis par une armée terroriste, une armée djihadiste contre la France») et de celles du président du principal parti d’opposition ("la guerre que nous devons livrer doit être totale»). Elle se voit confirmée aussi par la publication de la revendication de l'État islamique.

Cette primauté du cadrage relatif à la (situation de) guerre est cruciale dans l'organisation de tout le discours médiatique sur le 13 novembre, comme elle le fut déjà pour d'autres attentats9. Or, ce cadrage peut se discuter ou ne va pas de soi : au moment des attentats du 11-Septembre 2001, les médias américains se concentrent sur l'idée de "guerre» (Lewis, Reese, 2009), tandis que les médias européens n'en font pas un élément premier (Truc, 2016, p. 17-54). De même, une étude du traitement de quatre attentats majeurs survenus en 2004-2005 par quatre chaînes de télévision de pays différents (Gerhards, Schäfer, 2014) montre que deux d'entre elles développent la primauté de la «guerre», les autres insistant sur l'idée de «crime contre l'humanité ». Il n'y a pas en soi «la guerre » dans tout acte terroriste, mais une construction de sens plus ou moins disputée sur l'assimilation de cet acte à un acte de « guerre » ou de «crime de guerre » qui repose sur des mécanismes sémiotiques, lexicaux, discursifs, argumentatifs et normatifs.

Un discours «post-attentats » consisterait à poser que c'est parce qu'il y a de tels attentats que la France se retrouve soudainement en (situation de) guerre. C'est ce que suggère l'essentiel de la description des faits à mesure que leur gravité est attestée. Mais les interprétations convergent en même temps pour poser la relation inverse : c'est parce que la France est en (état de) guerre qu'elle subit ce type d'attentats dont la gravité ne pouvait donc qu'être anticipée.

\section{Dissensus/Consensus}

L'effet de sidération et l'injonction à l'unité nationale auraient-ils suspendu toute parole dissonante dans les premières heures du drame? L'expression, sinon d'un véritable dissensus, du moins de brèches dans le consensus attendu, se repère sur les plateaux télévisés dès le 13 novembre. On peut en distinguer deux formes : les dissensus d'expertise et les dissensus politiques.

9. Le concept de cadrage désigne les mécanismes narratifs par lesquels certains aspects d'une réalité perçue sont privilégiés au détriment d'autres possibles (Entman, 1993). Dans le cas du terrorisme, l'étude des discours médiatiques a ainsi été approfondie (Norris, Kern, Just éd., 2003; Papacharissi, Oliveira, 2008; Woods, 2011). 
Les dissensus d'expertise impliquent une situation langagière d'échange reposant sur une conflictualité entre différents discours de compétence. Peu nombreux et fugaces le 13 novembre, ils concernent avant tout les experts invités et les journalistes spécialisés. Ainsi, le chef du service Étranger de LCl exprime de manière tranchée son désaccord avec les propos tenus par un expert quelques minutes plus tôt, le désavouant sur les causalités géopolitiques avancées pour expliquer l'attentat :

Je crois qu'il ne faut pas tout mélanger, parler à la fois de l'Airbus qui a été abattu au-dessus des airs égyptiens, parler de l'attentat d'hier à Burj El Barajneh dans la banlieue sud de Beyrouth, ça n'a rien à voir même si c'est un djihad global, mondial [...]. Mais ça a pas grand-chose à voir. (TF1, 23h34)

Sur BFM TV, le consultant Police Dominique Rizet se fait le vecteur d'une critique externe qu'il reprend volontiers à son compte. Alors que le présentateur interprète le déplacement de François Hollande sur le site du Bataclan comme une preuve de la sécurisation du $11^{\mathrm{e}}$ arrondissement, D. Rizet se place du point de vue des forces de l'ordre, dont il est proche, et qui ont une lecture désapprobatrice de ce genre d'initiatives présidentielles :

Très franchement, dans la police on n'aime pas ça. On n'aime pas l'arrivée des autorités politiques sur place. Souvenez-vous, Jacques Chirac qui était allé dans le RER à Saint-Michel alors que les policiers étaient en train de travailler. [...] Imaginez qu'une camionnette soit garée dans la rue avec des explosifs... [...] Alors effectivement, l'hommage est important. Mais ça complique le travail de la police, disons-le clairement. (BFM TV, 1h31)

Ces dissensus d'expertise sont intéressants en tant qu'ils révèlent des cadres prédiscursifs collectifs et pointent ainsi des questionnements préfigurateurs de débats post-attentats, notamment sur les enjeux démocratiques et l'action publique. Sur BFM TV, l'ancien haut fonctionnaire de la Défense Pierre Conesa évoque ainsi un appareil militaire français doublement épuisé par les opérations extérieures et la mobilisation sur le territoire national, n'hésitant pas à dénoncer la passivité d'autres nations face à la situation syrienne. L'attente d'une production de sens dictée par le choc de l'événement autorise en quelque sorte l'expert à s'affranchir des précautions associées aux « connaissances tacites » du prédiscours (Paveau, 2006, p. 120) pour dire explicitement ce qui lui semble important d’être su pour le débat :

Dans notre opération en Syrie, l'Arabie Saoudite met à peu près autant d'avions que le Danemark et la Hollande réunis et nous on a quatre fois plus d'avions qu'eux. Par contre, ils ont quinze fois plus d'avions contre le Yémen. [...] Pour les djihadistes, nous faisons une croisade. J'attends le communiqué du roi d'Arabie Saoudite pour exprimer sa solidarité avec les Français [...]. Rappelons que l'Arabie Saoudite et les pays du Golfe n'accueillent aucun réfugié de ces crises. [...] La France et les pays occidentaux font une guerre qui n'est pas la leur. (BFM TV, 1h57) 
Sur France 2, la journaliste politique Nathalie Saint-Cricq explique avec gravité, tout en approuvant la mesure, ce que l'adoption de l'état d'urgence engendre pour la démocratie :

L'état d'urgence, c'est quelque chose d'extrêmement grave. On est à la limite de l'état de guerre. C'est-à-dire qu'il peut y avoir des libertés qui sont totalement entravées momentanément. (France 2, oh15)

Sans déroger à l'émotion collective qui domine le moment, l'expert pointe déjà des failles, souligne des effets pervers concernant le fonctionnement des politiques et des institutions. Ce faisant, il organise déjà la dynamique des débats post-attentats à venir sur des enjeux dépassant la perception subjective du drame qui se joue.

Plus structurels et récurrents sont les dissensus politiques. Dans les éditions spéciales, ils se déploient surtout le 14. Percevant la sphère politique comme l'espace des clivages, les journalistes ont tendance à marginaliser toute parole politique qui viendrait fissurer l'unité nationale attendue. Le registre discursif d'un discours médiatique appelant à l'unité est d'autant plus présent le 13 que pèse, une nouvelle fois en 2015, le spectre d'un acte terroriste commis par des membres de la communauté nationale (Garcin-Marrou, 2001). Au nom de cet impératif collectif, rares sont les responsables politiques à s'exprimer le soir des attentats (aucun sur France 2 et France 3, un sur TF1 et BFM TV). Quand ils le font, leurs transgressions verbales signent leur mise au silence. À 22 h56 sur BFM TV, quand Bernard Debré - qui devait intervenir sur la grève des médecins - évoque en creux le laxisme de François Hollande ("Tout le monde lui disait») et surtout qualifie avec certitude la nature de l'événement («Il s'agit à l'évidence d'attentats terroristes») au moment où les journalistes refusent encore d'authentifier celle-ci, le présentateur l'interrompt, appelle à la prudence («On a tous ce soir évidemment ce mot en tête qui n'est pour l'instant qu'une hypothèse ») et met fin à l'interview qui aura duré une minute!

Sur France 2, lorsque Nathalie Saint-Cricq annonce la décision des partis politiques de suspendre la campagne électorale en cours pour les régionales, elle manifeste simultanément la valeur dérisoire de cette information touchant pourtant son champ de compétence ordinaire («Alors je vais pas vous parler de politique intérieure parce que c'est vraiment ridicule ce soir») et formule la même injonction qui avait structuré les réactions après les attentats de janvier 2015 (Lefébure, Sécail éd., 2016) : «Dans ces moments-là, il n’y a pas de droite, de gauche. Voilà, il y a une espèce d'unité nationale et c'est le moins qu'on puisse en attendre ». Mais le lendemain, elle est déjà plus sceptique sur la capacité des responsables politiques à respecter cette ligne de conduite. Les clivages politiques n'ayant pas tardé à se manifester dans la matinée du 14, la journaliste laisse transparaître sa lassitude et formule un jugement sévère après les déclarations de Gilbert Collard appelant à la démission de François 
Hollande ou de Nicolas Sarkozy laissant entendre que l'arsenal policier et judiciaire est insuffisant :

C'est là qu'on va voir si on a une classe politique qui est à la hauteur des enjeux, qui est à la hauteur de la population française, [qui] réussit à s'en sortir sans qu'on retombe dans un certain nombre de bisbilles qui ne seraient pas du niveau. Et ça, on peut ne pas être totalement rassurés. (France 2, 14/11, 14h43)

En revanche, un consensus journalistique se dessine autour de la décision concrète de la soirée : l'adoption de l'état d'urgence dévoilée par François Hollande lors de sa première allocution. Dès lors, les journalistes, renouant avec leur fonction d'instance de validation ou d'invalidation de l'action politique, applaudissent la décision de fermeté du chef de l'État (tout en critiquant, comme sur TF1, l'ethos d'un président fébrile à la voix «chevrotante») :

La réponse est à la hauteur, on peut penser, de la vague d'attentats qu'il y a ce soir. (France 2, oh15)

Ce qui est nouveau, là, ce soir, dans la déclaration du président Hollande, qui est quand même assez extraordinaire, apparaissant en pleine nuit sur les écrans [...], c'est que, d'une part, il est dans le compassionnel, [...] et puis, vous avez des annonces qui montrent qu'il y a une véritable rupture : il y a la fermeture des frontières, il y a l'état d'urgence, mais il y a aussi la mobilisation de l'armée. (TF1, oho1)

La fermeté du chef de l'État est surtout saluée une heure plus tard, après sa deuxième allocution, sur le site du Bataclan (1h53) :

C'est vrai qu'il y avait beaucoup de sincérité dans le François Hollande de tout à l'heure. En même temps, on attend qu'il nous rassure [...] Donc, là, le chef de l'État, il est à sa place, dans son rôle [...] Avec des mots très forts : «combat impitoyable», «unie» et «déterminée». (TF1, 1h55)

Il a tenu à dire, dans ces circonstances-là, que les actes des terroristes ne seraient pas impunis et qu'ils trouveraient en face d'eux une République française déterminée [...] à les sanctionner comme on dit, ça va sans dire. Mais ça allait sans doute mieux en le disant ce soir. (BFM TV, 1h56)

Alimentés par les enquêtes d'opinion, les journalistes puisent à la source sondagière le moyen de conforter leur position et de repousser les débats clivants à venir sur l'état d'urgence :

Je pense que la population française est suffisamment mûre pour accepter [...] de subir un certain nombre de contraintes sans qu'il y ait une manifestation pour le côté liberticide et qu'on hurle contre cette sorte de couvre-feu. [...] Au vu des sondages précédents, les Français sont tout à fait prêts à jouer le jeu. Et puis, je vais vous dire, le choc est tel que, à mon avis, c'est pas le bon moment de hurler contre la police. (Nathalie Saint-Cricq, France 2, 14/11, 14h16) 
Les médias apparaissent ainsi bel et bien impliqués dans la logique d'action de l'État contre les menaces terroristes. Parvenant difficilement à construire des cadres discursifs autonomes et à s'extraire d'une logique de coproduction du discours avec les autorités (Garcin-Marrou, 2001), ils participent à la promotion d'une perspective institutionnelle de la communauté nationale (Matthews, 2016).

L'étude des premières heures qui suivent un attentat majeur ne suffit pas à identifier tous les discours qu'il générera. Mais, comme cela a pu être mis au jour à propos de l'analogie entre les attentats du 11-Septembre et l'attaque japonaise de Pearl Harbor durant la Seconde Guerre mondiale (Chéroux, 2009, p. 55-60; Truc, 2016, p. 23-32), il se confirme que des éléments importants s'organisent dans cette temporalité très resserrée et qu'ils exercent une influence durable sur l'orientation des récits à venir. Concernant le suivi télévisé en direct, et d'une manière assez similaire à ce qui a pu être identifié pour les attentats du 11-Septembre (Lamy, 2006), deux perspectives narratives viennent compléter le régime discursif journalistique de restitution d'une factualité encore incertaine. Il s'agit, d'une part, de l'injonction à éprouver les événements de manière intersubjective (rapport aux témoins) et, d'autre part, de l'injonction à faire sens des événements par un travail très précoce d'interprétation (rapport aux experts). Le traitement médiatique des premières heures consiste en un entrelacement de diverses dimensions du rapport aux faits qui en rend l'appréhension encore plus complexe en termes de réception. Les aspects émotionnels, cognitifs et normatifs sont ainsi sollicités par alternance de séquences plus ou moins courtes qui composent par accumulation le temps resserré des éditions spéciales.

Étudier ces premiers moments permet aussi d'établir à quel point se trouvent mobilisés un vocabulaire de dénomination, des perspectives d'interprétation et des formes de jugement inscrites dans un socle déjà disponible (prédiscours), plutôt que ne surgit un ordre discursif propre à l'événement. C'est ainsi l'insertion de l'attentat dans ses contextes institutionnel, culturel et politique qui apparaît comme un enjeu que l'analyse de discours peut éclairer. À cet égard, un élément important distingue le traitement des attentats du 11-Septembre aux États-Unis de ceux du 13 novembre en France : si l'on peut relever à propos des premiers un champ lexical de l'inattendu (Lamy, 2006) - « inimaginable», «irréel»-, celui-ci n'apparaît jamais pour les seconds qui sont au contraire, dans un monde occidental marqué depuis une quinzaine d'années par la répétition de massacres terroristes d'inspiration islamiste, systématiquement évoqués comme présumés devoir se produire aussi en France. Dans les discours de description et d'interprétation, l'exceptionnalité des faits s'articule donc à leur intégration dans un contexte bien installé et identifié qu'ils viennent d'ailleurs confirmer.

Cette dialectique de l'exceptionnalité non pas en rupture avec le monde vécu (tel que le sentiment de basculement d'un monde à l'autre dans le suivi 
du 11-Septembre) mais comme expression (enfin actualisée) du monde vécu est une caractéristique majeure de la mise en discours médiatique des attentats du 13 novembre 2015. Il s'avère qu'elle procède essentiellement de la prétention à la juste interprétation des journalistes spécialisés et des experts qui sont des figures d'énonciation mobilisées dans le cadre du studio télévisé. Par comparaison avec les éléments factuels énoncés par les reporters de terrain, qui permettent d'attester que quelque chose se passe, le studio s'avère être le dispositif d’édification du sens. La «vérité » élucidante ou interprétative du studio vient donc en relais de la "vérité» factuelle, mais la supplante en termes de tangibilité et de rapport à l'événement au fur et à mesure que les heures passent, dès la nuit des attentats.

Ce constat rejoint celui effectué sur les mécanismes du discours d’information télévisé (Brusini, James, 1982). La "télévision d'enquête» des années 1950-1960, où «le journalisme fait du reporter un témoin professionnel et du témoignage un moyen d’informer » (p. 68), est supplantée à partir des années 1970 par une «télévision d'examen », où la source visuelle extraite du terrain fournit l'opportunité de traiter d'un thème depuis le studio, où le discours a plus de valeur que l'image. Le studio n'est alors plus un espace d'exposition ou de restitution mais un lieu-fonction, véritable dispositif de production de sens par la valeur des énonciateurs qui y sont mobilisés et par l'utilité des discours qu'ils énoncent (le cas échéant de manière contradictoire). La mobilisation de la figure de l'expert, adoptée par les chaînes d'information en continu depuis le courant des années 2000, contribue au déploiement de cette « télévision d'examen » dont il convient cependant d'assumer qu'elle s'articule au paradigme d'authentification de la «télévision d'enquête». Dans les circonstances spécifiques des attentats du 13 novembre, dont la médiatisation reste marquée par l'absence générale d'images en provenance des lieux attaqués eux-mêmes (Aubert, Charaudeau, Mehl, 2018), ce mécanisme s’observe clairement.

Il importe enfin de considérer les conditions matérielles et organisationnelles du travail médiatique pour distinguer les aspects propres à chaque chaîne des aspects qui leur sont communs. Les caractéristiques partagées que nous avons identifiées construisent une mise en récit du 13 novembre assurant trois opérations complémentaires. D'abord, la mise en récit rapporte l'événement à d'autres (sérialisation) de manière à ce qu'il concerne toute la collectivité dans sa continuité, même si les perceptions des individus peuvent varier. Ensuite, si la mise en récit s'attache aux caractéristiques exceptionnelles de l'événement, elle l’interprète comme le révélateur d'un état préalable dont il est un symptôme (interprétation causale). Enfin, tout en intégrant techniquement de savantes divergences de description ou d'interprétation (dissensus), la mise en récit analytique en studio promeut normativement l'unité du collectif (national) touché par l'attentat (consensus). 


\section{Références}

Altheide David L., 2017, Terrorism and the Politics of Fear, Lanham, Rowman \& Littlefield.

- 2009, Terror Post $9 / 11$ and the Media, New York, Peter Lang.

ARQUEMBOURG Jocelyne, 2003, Le temps des événements médiatiques, Paris, Bruxelles, INA, De Boeck.

Aubert Aurélie, Charaudeau Patrick, Mehl Dominique, 2018, "Les attentats du 13 novembre sur BFM TV : informer en direct face au défi terroriste », Réseaux, n²07, p. 229-254.

BLoNDHEIM Menahem, LIEBES Tamar, 2003, «From disaster marathon to media event: live television's performance on September 11, 2001, and September 11, 2002 ", dans Crisis Communications: Lessons from September 11, A. M. Noll éd., Lanham, Rowman \& Littlefield, p. 185-197.

BRUSINI Hervé, JAMES Francis, 1982, Voir la vérité : le journalisme de télévision, Paris, PUF.

CALABRESE SteImberg Laura, 2012, "L'acte de nommer : nouvelles perspectives pour le discours médiatique», Langage et société, nº140, p. 29-40.

CHÉroux Clément, 2009, Diplopie. L'image photographique à l'ère des médias globalisés : essai sur le 11 septembre 2001, Cherbourg, Le Point du Jour.

ENTMAN Robert, 1993, «Framing: toward clarification of a fractured paradigm », Journal of Communication, vol.XLIII, nº 4 , p. 51-58.

Frau-Meigs Divina, 2005, Qui a détourné le 11 septembre? Journalisme, information et démocratie aux États-Unis, Bruxelles, De Boeck.

Garcin-Marrou Isabelle, 2001, Terrorisme, médias et démocratie, Lyon, Presses universitaires de Lyon.

GERHARDS Jürgen, SCHÄFER Mike S., 2014, «International terrorism, domestic coverage? How terrorist attacks are presented in the news of CNN, Al Jazeera, the BBC, and ARD », The International Communication Gazette, vol. LXXVI, nº 1, p. 3-26.

KRIEg-PlANQUe Alice, 2009, "À propos des “noms propres d'événement” : événementialité et discursivité », Les Carnets du Cediscor, n 11, p. 77-90.

- 2003, "Purification ethnique»: une formule et son histoire, Paris, CNRS Éditions.

LAMY Aurélia, 2006, «Les spécificités du traitement médiatique dans l'urgence : l'exemple des attentats du 11 septembre 2001», Communication \& Organisation, n 29, p. 108-122.

LefÉbuRe Pierre, SÉCAIL Claire éd., 2016, Le défi Charlie : les médias à l'épreuve des attentats, Paris, Lemieux éditeur.

LEWIS Seth C., REeSE Stephen D., 2009, «What is the war on terror? Framing through the eyes of journalists », Journalism \& Mass Communication Quarterly, vol. LXXXVI, n० 1 , p. 85-102.

MATTHEWS Julian, 2016, «Media performance in the aftermath of terror: reporting templates, political ritual and the UK press coverage of the London Bombings, 2005 ", Journalism, vol.XVII, n² 2, p. 173-189.

MoIRAnd Sophie, Rebou L-Touré Sandrine, 2015, « Nommer les événements à l'épreuve des mots et de la construction du discours ", Langue française, n ${ }^{0} 188$, p. 105-120.

NorRIS Pippa, Kern Montague, Just Marion éd., 2003, Framing Terrorism: The News Media, the Government, and the Public, New York, Routledge.

PAPACHARISSI Zizi, OLIVEIRA Maria de Fatima, 2008, « News frames terrorism: a comparative analysis of frames employed in terrorism coverage in U.S. and U.K. newspapers », The International Journal of Press/Politics, vol. XIII, nº 1, p. 52-74. 
Paveau Marie-Anne, 2006, Les prédiscours : sens, mémoire, cognition, Paris, Presses Sorbonne Nouvelle.

SCANNELL Paddy, 2006, "Quelle est la réalité du malheur?», dans La terreur spectacle: terrorisme et télévision, D. Dayan éd., Bruxelles, De Boeck, p. 25-39.

TRUc Gérôme, 2016, Sidérations : une sociologie des attentats, Paris, PUF.

Woods Joshua, 2011, «Framing terror: an experimental framing effects study of the perceived threat of terrorism », Critical Studies on Terrorism, vol. IV, nº 2, p. 199-217.

\section{Résumé / Abstract / Compendio}

\section{Les attentats du 13 novembre en direct à la télévision : mise en récit de l'évé- nement et de ses ramifications}

À partir d'une analyse de contenu et de discours des éditions télévisées spéciales des 13 et 14 novembre 2015, cet article analyse comment l'hétérogénéité des mises en récit de l'événement terroriste façonne des cadres interprétatifs distincts. Intégrant en partie des phénomènes prédiscursifs, les discours énoncés en direct contiennent les sédiments de récits antérieurs et à venir. L'étude de cet ensemble discursif invite à s'interroger sur la définition d'une société «post-attentats».

Mots-clés: attentats du 13 novembre 2015, télévision, information, terrorisme, discours médiatique

\section{The November $13^{\text {th }}$ terror attacks live on television: the narratives of an event and its ramifications}

Based on a contents and discourse analysis of November 13 and 14, 2015 TV "breaking news" programs, this article analyzes how the heterogeneity of TV discourse about the terror event shapes distinct narrative schemes. Since live stories and comments partly rest on prediscursive processes, they include traces of past and future narratives, thus showing to what extent the French society can conditionally be conceived as a "postattack" society.

Keywords: November 13 attacks, television, information, terrorism, media narratives

\section{Los atentados del 13 de noviembre en vivo en la televisión: las narraciones del evento y de sus ramificaciones}

Basado en un análisis de contenido de ediciones especiales de televisión del 13 y 14 de noviembre de 2015 , este artículo analiza cómo la heterogeneidad de la narración del evento terrorista forma distintos marcos interpretativos. Integrando parcialmente fenómenos prediscursivos, los discursos enunciados en vivo contienen los sedimentos de relatos pasados y futuros. El análisis de este discursos nos invita a cuestionar la definición de una sociedad «post-atentado».

Palabras claves: atentado del 13-Noviembre, televisión, información, terrorismo, discurso mediático 